\title{
EUROPEAN ORGANIZATION FOR NUCLEAR RESEARCH
}

CERN - AB Division

CERN-AB-2003-020 ABP

CLIC Note 564

Nonlinear Optimization of a Low Emittance CLIC Damping Ring Lattice

M. Korostelev and F. Zimmermann, CERN

\begin{abstract}
The CLIC damping ring design is optimized to produce a beam with ultra low emittances. The lattice for such a machine requires a small value of the optical functions, a large number of compact arc cells and, for the chromatic correction, strong sextupoles, that introduce significant nonlinearities, decreasing the dynamic aperture. In this paper, the nonlinear optimization of the damping ring lattice is described.
\end{abstract}

\author{
Presented at PAC 2003, Portland, Oregon, USA \\ from 12 to 16 May 2003
}

Geneva, Switzerland

22 May 2003 


\title{
Nonlinear Optimization of a Low Emittance CLIC Damping Ring Lattice
}

\author{
Maxim Korostelev and Frank Zimmermann \\ CERN, Geneva, Switzerland
}

\section{Abstract}

The CLIC damping ring design is optimized to produce a beam with ultra low emittances. The lattice for such a machine requires a small value of the optical functions, a large number of compact arc cells and, for the chromatic correction, strong sextupoles, that introduce significant nonlinearities, decreasing the dynamic aperture. In this paper, the nonlinear optimization of the damping ring lattice is described.

\section{CLIC LATTICE DESIGN}

The Theoretical Minimum Emittance (TME) lattice [2, $3,4,5,6,8,9]$ is most suitable for the low emittance compact arc cell needed for the damping ring of CLIC [1]. The length of a cell is important since the damping time is directly proportional to the ring circumference. Comparing the minimum emittance of the TME lattice with the minimum emittances produced by three other lattice types (Double Focusing Achromat, Triplet Achromat Lattice, Triple Bend Achromat) [10] for a fixed bending angle $\theta$, we obtain the following ratios:

$$
\frac{\varepsilon_{D F A}^{m i n}}{\varepsilon_{T M E}^{m i n}}=3, \quad \frac{\varepsilon_{T A L}^{m i n}}{\varepsilon_{T M E}^{m i n}}=12, \quad \frac{\varepsilon_{T B A}^{m i n}}{\varepsilon_{T M E}^{m i n}}=\frac{7}{3} .
$$

The energy for the CLIC damping ring was chosen as $2.42 \mathrm{GeV}$. At lower energy (for example $1.98 \mathrm{GeV}$ ), the intrabeam scattering (IBS) is much stronger. The IBS growth time is proportional to $\gamma^{6}$, while the damping time is inversely proportional to $\gamma^{3}$.

A TME arc cell of the CLIC damping ring comprises four quadrupoles and a combined function bending magnet. The two arcs of the ring are connected by long dispersionfree straight sections that include RF cavities, FODO cells with damping wigglers, and injection/extraction sections. The design parameters of the damping ring are presented in Table 1.

At the exit of the main linac, the horizontal emittance $\gamma \varepsilon_{x}$ should not exceed $680 \mathrm{~nm}$, and the vertical emittance $\gamma \epsilon_{y}$ be smaller than $10 \mathrm{~nm}$. However, downstream of the damping ring some additional emittance dilutions are expected both in the linac and in the bunch compressors.

The values of the equilibrium transverse beam emittances $\varepsilon_{x}, \varepsilon_{y}$, rms energy spread $\sigma_{\delta}$, and rms bunch length $\sigma_{s}$ were computed for the proposed damping ring design by a step-wise integration in time, using the Bjorken-Mtingwa formalism [11] for the IBS growth rates.

\section{LINEAR AND NONLINEAR PROPERTIES}

\section{$2.1-I$ Transformer}

Sextupoles introduce both second order geometric aberrations and chromatic aberrations. If two thin-lens sex-
Table 1: List of CLIC damping ring parameters.

\begin{tabular}{lcc}
\hline Parameter & Symbol & Value \\
\hline Nominal ring energy & $\gamma m c^{2}$ & 2.424 \\
No. of bunche trains stored & $N_{\text {train }}$ & 9 \\
Ring circumference & $C$ & $357.2[\mathrm{~m}]$ \\
Number of cells & $N_{\text {cells }}$ & 96 \\
Extracted hor. emittance at IBS & $\gamma \varepsilon_{x}$ & $620[\mathrm{~nm}]$ \\
Extracted vert. emittance at IBS & $\gamma \varepsilon_{y}$ & $8.7[\mathrm{~nm}]$ \\
Extracted long. emittance at IBS & $\gamma m c^{2} \varepsilon_{t}$ & $4319[\mathrm{eV} \times \mathrm{m}]$ \\
Extracted energy spread at IBS & $\sigma_{\delta}$ & $1.36 \times 10^{-3}$ \\
H. betatron tune & $Q_{x}$ & 72.85 \\
V. betatron tune & $Q_{y}$ & 34.82 \\
Betatron coupling & $\varepsilon_{y 0} / \varepsilon_{x 0}$ & $2.1 \%$ \\
Field of bending magnet & $B_{a}$ & $9.32[\mathrm{kG}]$ \\
Length of bending magnet & $L$ & $0.545[\mathrm{~m}]$ \\
Phase advance per arc cell & $\mu_{x} / \mu_{y}$ & $210^{\circ} / 90^{\circ}$ \\
Field of wiggler & $B_{w}$ & $17.64[\mathrm{kG}]$ \\
Momentum compaction & $\alpha_{p}$ & $0.731 \times 10^{-4}$ \\
Energy loss per turn & $U_{0}$ & $2.1916[\mathrm{MeV}]$ \\
RF frequency & $f_{r f}$ & $1500[\mathrm{HHz}]$ \\
RF voltage & $V_{m}$ & $3.0[\mathrm{MV}]$ \\
Revolution time & $T_{r}$ & $1.191[\mu s]$ \\
Harmonic number & $h$ & 1786 \\
\hline
\end{tabular}

tupoles of equal strength are placed at the entrance and exit of a $-I$ transformer the geometric aberrations introduced by the two sextupoles exactly cancel each other. A perfect cancellation of the geometric aberrations produced by the sextupoles would be to place separate $-I$ transformers of sextupole pairs in such a way that they do not interfer with each other. In reality, the finite length of the sextupoles compromises this cancellation. For example, consider the 1-D motion through a pair of thick sextupoles both of strength $S$ and length $L$, separated by a $-I$ transformer between the entrance of the first and the second sextupole. In Lie algebra notation, the map of the complete two-sextupole system is

$$
M=e^{: f:} e^{:-\frac{1}{2} L x^{\prime 2}: E}
$$

where $f$ denotes the linear $(-I)$ transformation between the entrances of the two sextupoles. The error map $E$ is

$$
E=e^{: \frac{1}{6} S^{2} L^{3} x^{4}+O\left(L^{4}\right)}
$$

The dominating term in the error map is of the order $O\left(L^{3}\right)$, and it looks like an octupole term.

\subsection{Second-Order Achromat}

In our case, non-interlaced $-I$ transformers with thin sextupoles are impossible to realize, because there is not 


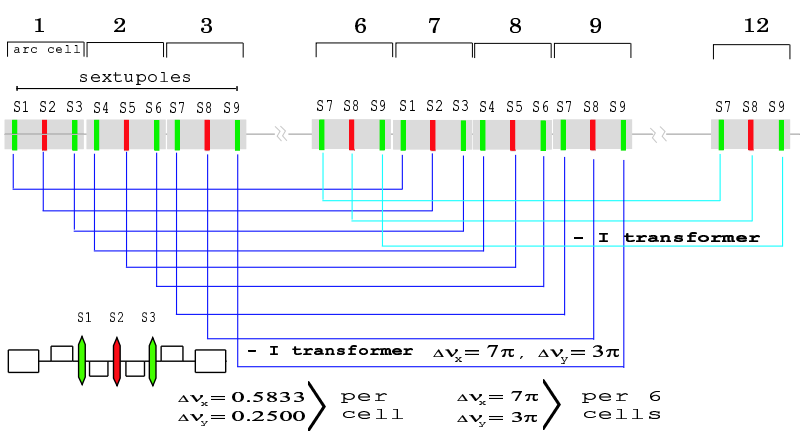

Figure 1: Second-order achromat of the damping ring.

enough space available to arrange them. The small beta and dispersion functions require a sufficient number of strong sextupoles in order to correct the large horizonal and vertical chromaticities.

According to Brown's 1st theorem $[12,13]$, if a lattice consists of $n$ identical cells with $n>3$ and has a total phase advance $2 \pi m$, all second-order geometric aberrations are cancelled. The uncorrected geometric aberrations introduced by the crosstalk of interlaced $-I$ sextupolar transformers are of third and higher order.

In the proposed damping ring design [9], interlaced sextupole pairs are used for the chromatic correction. Nine families of sextupoles were chosen, located in 3 adjacent arc cells, as it is shown in Fig. 1 (three families for the vertical motion and six families for the horizontal motion).

The damping ring comprises two arcs, each of which consists of 48 cells. At the given number of TME arc cells, it is possible to tune the phase advance of the cell from $180^{\circ}$ degree to a maximum value of $284^{\circ}$ degree, keeping the cell length less than $2.5 \mathrm{~m}$. Taking into account IBS, a larger phase advance per cell reduces the final emittances and also the average values of betatron and dispersion function over the cell. But it increases the strength of the sextupoles, which induces strong nonlinearities and consequently limits the dynamic aperture. Tuning the phase advance by variation of quadrupoles strength, drift length, and gradient field of bending magnet, the natural chromaticities can be minimized. However, to achieve a sufficient beta split at the location of the sextupoles is complicated, due the required short length of the arc cell.

The horizontal and vertical phase advances per cell were chosen as $210^{\circ}$ and $90^{\circ}$ degree, respectively. Such phase advances meet second-order achromat requirements and also provide acceptable emittances. Three adjacent arc cells form a super-period. By imposing the $-I$ transformation conditions over 6 cells (cancellation between first and seventh cell, 2nd and 8th, 3rd and 9th, 6th with 12th, etc.) for both vertical and horizontal motion, the total phase advance over four identical super-period cells is a multiple of $2 \pi$. The $X$ and $Y$ phase advances over 12 arc cells with repetitive symmetry are $7 \times 2 \pi$ and $3 \times 2 \pi$, respectively, that is a first order transfer matrix equal to unity in both transverse planes. Thus, a second-order achromat using the interlaced $-I$ transformers is assembled, which provides a cancellation of all second-order geometric aberrations.

\subsection{Chromatic Correction}

The first and second order chromatic terms are corrected by matching the strengths of 9 sextupole families. Without chromatic correction the natural first order chromaticities $\xi_{x}$ and $\xi_{y}$ are -115 and -124 for the horizontal and vertical plane, respectively. The chromaticity contribution of the two very long straight sections is $\Delta \xi_{x} \approx 23 \%$ and $\Delta \xi_{x} \approx 30 \%$.

The D-sextupole is placed between the two identical defocusing quadrupoles where the beta split is maximum. There are only two possible location of F-sextupoles for the proposed arc cell. First position is between the bending magnet and the focusing quadrupole. The second one is between the focusing and defocusing quadrupoles. At the first option, the strength of F and D sextupoles is $32 \%$ and $25 \%$ respectively less than in second option. However, the

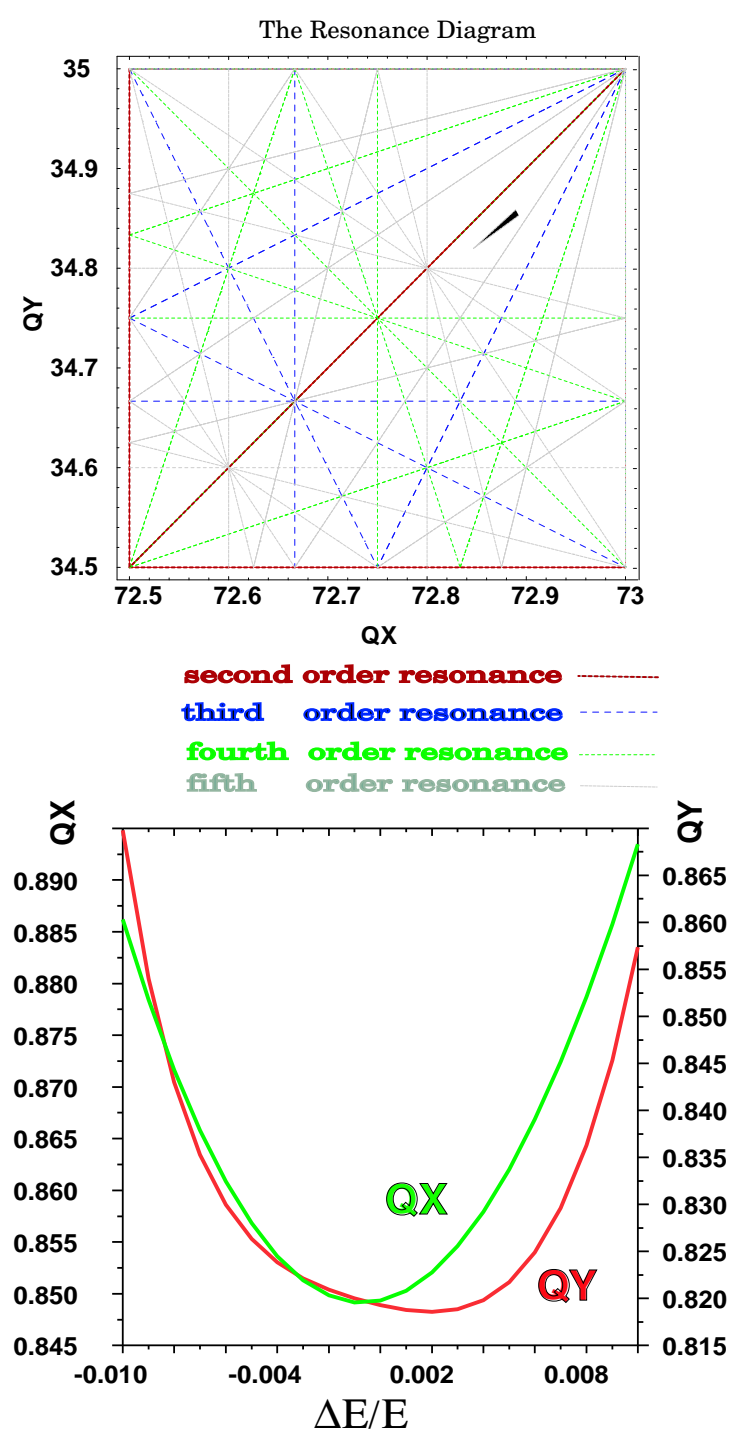

Figure 2: The tune shift versus momentum deviation (bottom) and the same on a resonance diagram (top). 
first option of sextupole placement gives significant tune shift with amplitude that limits dynamic aperture. Thus, the second option was chosen.

In fact, the strengths of all horizontal sextupole families are nearly equal, with a difference of less than one percent. The same is true for the vertical families. In the case of only two families, the second order chromatic aberrations for the vertical and horizontal motion are not exactly equal to zero, but $\partial^{2} Q_{x} / \partial \delta^{2}=16$ and $\partial^{2} Q_{y} / \partial \delta^{2}=143$ still not significantly contributing to the tune shift with momentum deviation.

The strengths of the chromatic sextupoles are considerable (but their pole-tip fields do not exceed a value of $2 \mathrm{~T}$, which we considered a reasonable upper bound). The tune shift over a large momentum range of $\pm 1 \%$ is shown in Fig. 2. A low periodicity of the damping ring results from the two long straight sections. The working point for zero momentum offset was chosen as $Q_{x} / Q_{y} \rightarrow 0.85 / 0.82$. The working point can be shifted by changing the FODO cell phase advance, while the arc phase advance should be kept fixed.

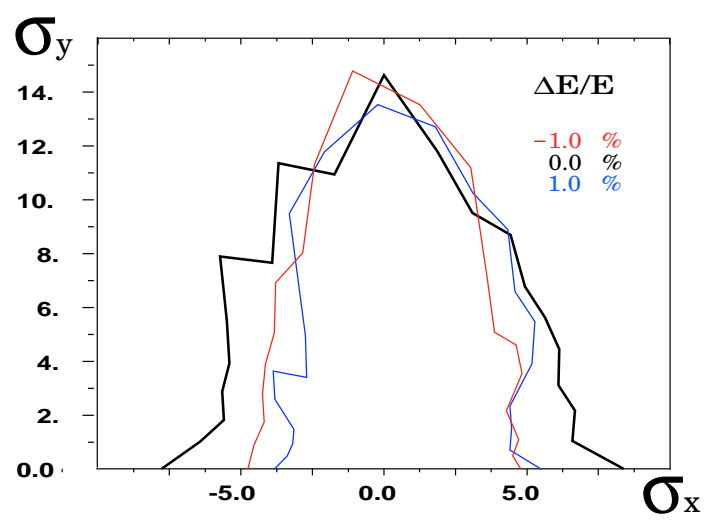

Figure 3: The dynamic aperture of the injected beam, as determined by particle tracking over 1000 turns.

\subsection{Dynamic Aperture}

To enhance the dynamic aperture, harmonic sextupoles can be installed in the non-dispersive sections[14]. In our present lattice, two families of harmonic sextupoles are used. Each consists of eight sextupoles, which are located in the wiggler sections. By means of the harmonic sextupoles, the dynamic aperture is increased about two times, so that its value now corresponds to about six rms beam sizes for an on-momentum particle, as it is shown in Fig. 9. Strengths and positions of the harmonic sextupoles were chosen empirically by particle tracking. The dynamic aperture might be further improved by increasing the number of harmonic-sextupole families and, possibly, by adding some octupole correctors which may cancel third-order terms resulting from crosstalk.

\section{OUTLOOK}

The final equilibrium emittances obtained by the present damping-ring lattice have been estimated via numerical in- tegration. The longitudinal emittance is $5 \%$ below the specification. The two transverse emittances are smaller than those required at the end of the CLIC main linacs, but the horizontal emittance exceeds the original target value [1] by $38 \%$ (620 nm compared $450 \mathrm{~nm}$ ), which is due to IBS.

Recently, the dynamic aperture of the ring has been optimized by means of 9 families of chromatic sextupoles, consisting of interleaved $-I$ pairs, and 2 additional harmonic families. It now exceeds 5-6 rms beam sizes at injection. The momentum acceptance of the lattice surpasses $\pm 1 \%$.

In the future, we plan to investigate the following three items: (1) the effect of alignment errors and beam-based tuning on the dynamic aperture, (2) the optimum choice of the emittance ratio, representing linear betatron coupling between the horizontal and vertical plane, which is an external input to the emittance computation, and (3) the potential for a transverse emittance reduction by increasing the length of the wiggler.

\section{REFERENCES}

[1] R. Assmann et al., G. Guignard (ed.), "A 3-TeV e $\mathrm{e}^{-}$Linear Collider Based on CLIC Technology," CERN-2000-008 (2000).

[2] L. Teng, "Minimizing the Emittance in Designing the Lattice of an Electron Storage Ring," FERMILAB-TM-1269 (1984).

[3] L. Teng, S.Y. Lee, "Theoretical Minimum Emittance Lattice for an Electron Storage Ring," (1991).

[4] J.P. Potier, L. Rivkin, "A Low Emittance Lattice for the CLIC Damping Ring,” CERN-PS-97-020 (1997).

[5] J.P. Potier, T. Risselada, "Fundamental Design Principles of Linear Collider Damping Rings, with Application to CLIC,", EPAC 2000, Vienna, CERN-PS-2000-037-LP, CLIC-NOTE-439 (2000).

[6] P. Emma, T. Raubenheimer, "Systematic Approach to Damping Ring Design," Phys. Rev. ST Accel. Beams 4, 021001 (2001).

[7] H. Wiedemann, "Low Emittance Storage Ring Design,'Lecture Notes in Physics 296, Texas (1986).

[8] J.M. Jowett, T. Risselada, F. Zimmermann, H. Owen "Damping Rings for CLIC," PAC2001, Chicago (2001).

[9] M. Korostelev, F. Zimmermann, "Optimization of CLIC Damping Ring Design Parameters," EPAC 2002 Paris (2002).

[10] A. Ropert, "High Brilliance lattice and the effects of ID," CERN Accelerator School, 6-13 April (1989).

[11] J.D. Bjorken, S.K. Mtingwa, "Intrabeam Scattering," Part. Accel. 13, 115-143 (1983).

[12] K. Brown, "A Second Order Magnetic Optical Achromat," SLAC-PUB-2257 (1979).

[13] K. Brown, R. Servrancks, "Applications of the SecondOrder Achromat Concept to the Design of Particle Accelerators," Proc. PAC95, p. 2288 (1995).

[14] A. Wolski, "Optimisation Of The Dynamic Aperture Of The DIAMOND Storage Ring Lattice," EPAC 2000 Vienna (2000). 